\title{
EDITORIAL \\ Spinal infections: a growing problem, which deserves our urgent attention
}

\author{
Jens R. Chapman, MD \\ Department of Orthopedics, Swedish Neuroscience Institute, Seattle, Washington
}

$\mathrm{S}$ PINAL infections are indeed a growing problem, which in this surgeon's lifetime has changed from an occasional occurrence to a literal weekly clinical encounter. ${ }^{3}$ The spinal column is a target organ for opportunistic infections with its significant vascular perfusion system and its many end capillaries and valves. ${ }^{6}$ Changing antibiotic resistance patterns and a decreasing general health status may all play a significant role in this clinical problem. Undoubtedly, there are significant consequences to this still-evolving development; for one, the cost component with its diagnostic, medical, as well as surgical therapies and lost productivity time remains significant but has not yet been adequately quantified. Currently there is not even agreement on how long to perform what type of antibiotic therapy. From a patient perspective, delays in diagnosis and providing adequate care can lead to significant disability or even death. ${ }^{7}$ Guidelines for diagnosis and treatment of de novo spine infections are still absent or woefully imprecise due to lack of larger supportive studies. ${ }^{4}$ This Neurosurgical Focus issue on spinal infections deserves credit for highlighting the many still-unresolved questions that plague clinicians. ${ }^{2}$

The study by Behmanesh et al. on the subject of using echocardiography as a routine diagnostic tool for inpatients with de novo spinal infections is important because it underscores the multisystem nature of these infections and the susceptibility of affected patients to become much more ill or be undertreated if not addressed adequately. ${ }^{2}$ The authors make a convincing argument that transesophageal echocardiography (TEE) detects infective endocarditis (IE) with much higher specificity than detected by intuitive care management in a historical control group (33\% vs $3 \%$ ) while showing no loss of sensitivity. While their results make intuitive sense, there are several questions raised by this study. Aside from the usual concerns about the relevance of historical control groups in times of rapidly changing medical standards of practice and technology, the main issues remain as follows.

First, the authors did not report on the outcomes of their historical control group. Did they experience more organ failures, spinal disease recurrence, or even death than the more recent study group that received TEE? Note that intravenous drug use in both of their study groups was $1.4 \%$ and $5.6 \%$, respectively; it stands to reason that the rate of intravenous drug users would be much higher in other regions and cultures.

Second, the authors also decided on using TEE over the more conventional transthoracic noninvasive echocardiogram variant. They stated a higher sensitivity of TEE over transthoracic echocardiography as justification for their diagnostic modality decision, yet offer only a single reference from 1997 in support of their contention. Surely ultrasound technology has advanced in leaps and bounds since that time, and the choice of a noninvasive technology over an invasive test would certainly hold some appeal. ${ }^{5}$

These methodological criticisms should not detract from the general message of the study and the authors' work, which clearly identifies a substantial incidence of patients (33\%) with de novo spine infections having infections of their valves and endocardium. Identifying and addressing these pathological entities early in the treatment process appears to be a clear and reasonable suggestion for future workups.

As we move forward it would probably be helpful to better differentiate infection types not just by microorganisms, but also by various general host comorbidities, which could be contributory to the disease propagation. From a spine standpoint our differentiation would benefit from differentiating pure epidural abscesses from discitis and the discitis/osteomyelitis with instability. It would be desirable to include the neurological status referable to the infectious process (intact, incomplete, or complete) 
and their general localization (cervical, thoracic, lumbar, or multifocal) as well. ${ }^{1,7}$ Along with better classifications of spinal infections and a more consistent application of diagnostic algorithms, including severity of the inflammatory response, nutritional status, and spinal stability, the assessment of the cardiac system for colonization with some form of echocardiogram appears to be a medically sensible addition. To this end, getting well-thoughtthrough diagnostic algorithms for diagnosis and therapy of de novo spine infections into our electronic medical records systems seems to be a long overdue step. This article by Behmanesh et al. makes a cogent argument despite its acknowledged shortcomings, and is a clear step in the right direction. ${ }^{2}$

https://thejns.org/doi/abs/10.3171/2018.10.FOCUS18587

\section{References}

1. Alton TB, Patel AR, Bransford RJ, Lee MJ, Bellabarba CB, Chapman JR: Is there a difference in neurologic outcome in medical versus early operative management of cervical epidural abscesses? Spine J 15:10-17, 2015

2. Behmanesh B, Gessler F, Schnoes K, Dubinski D, Won SY, Konczalla J, et al: Infective endocarditis in patients with pyogenic spondylodiscitis: implications for diagnosis and therapy. Neurosurg Focus 46(1):E2, 2019

3. Blecher R, Yilmaz E, Drazin D, Oskouian RJ, Chapman Jens R: Recent increase in the rate of spinal infections may be related to growing substance-use disorder in the state of
Washington: wide population-based analysis of the Comprehensive Hospital Abstract Reporting System (CHARS) database. Spine (Phila Pa 1976) [epub ahead of print], 2018

4. Farber SH, Murphy KR, Suryadevaraa CM, Babu R, Yang S, Feng L, et al: Comparing outcomes of early, late, and nonsurgical management of intraspinal abscess. J Clin Neurosci 36:64-71, 2017

5. Fowler VG, Li J, Corey GR, Boley J, Marr KA, Gopal AK, et al: Role of echocardiography in evaluation of patients with Staphylococcus aureus bacteremia: experience in 103 patients. J Am Coll Cardiol 30:1072-1078, 1997

6. Griessenauer CJ, Raborn J, Foreman P, Shoja MM, Loukas M, Tubbs RS: Venous drainage of the spine and spinal cord: a comprehensive review of its history, embryology, anatomy, physiology, and pathology. Clin Anat 28:75-87, 2015

7. Patel AR, Alton TB, Bransford RJ, Lee MJ, Bellabarba CB, Chapman JR: Spinal epidural abscesses: risk factors, medical versus surgical management, a retrospective review of 128 cases. Spine J 14:326-330, 2014

\section{Disclosures}

The author reports no conflict of interest.

\section{Correspondence}

Jens R. Chapman: jens.chapman@swedish.org.

INCLUDE WHEN CITING

DOI: $10.3171 / 2018.10 . F O C U S 18587$. 